\title{
Poincaré Gauge Theory and Galilean Covariance
}

\author{
M. de Montigny, ${ }^{1,2}$ F. C. Khanna, ${ }^{2,4}$ A. E. Santana, ${ }^{3}$ E. S. Santos, ${ }^{3}$ \\ and J. D. M. Vianna ${ }^{3,5}$
}

${ }^{1}$ Faculty St. Jean, University of Alberta, 8406-91 Street, Edmonton, Alberta T6C 4G9, Canada;

${ }^{2}$ Theoretical Physics Institute, Department of Physics, University of Alberta, Edmonton, Alberta T6G 2J1, Canada;

${ }^{3}$ Instituto de Física, Universidade Federal da Bahia, Campus de Ondina, 40210-340, Salvador, Bahia, Brazil;

${ }^{4}$ TRIUMF, 4004, Wesbrook Mall, V6T 2A3, Vancouver, British Columbia, Canada; and

${ }^{5}$ Instituto de Física, Universidade de Brasília, 70910, Brasília, DF, Brazil

Received June 16, 1999

We analyse the Poincare gauge structure proposed by D. Cangemi and R. Jackiw (CJ) (Ann. Phys. (N.Y.) 225, 229), in association with general Lie algebras and, in particular, with Galilean symmetries. In this context, the CJ method is formulated as an embedding scheme of metric spaces, and aspects of Galilean covariance are then used to analyse: (i) the nonrelativistic limits of the electromagnetic field; (ii) a Galilean counterpart of the CJ theory; (iii) geometrical common structures of Lorentzian and Galilean physics; and (iv) a covariant formalism for classical mechanics. (C) 1999 Academic Press

\section{INTRODUCTION}

Recently, Cangemi and Jackiw $(\mathrm{CJ})[1-3]$ described $(1+1)$-dimensional gravity as a gauge theory in which the gauge group is a central extension of the $(1+1)$ dimensional Poincaré group

$$
[P, H]=I, \quad[K, H]=P, \quad[K, P]=H,
$$

where, as usual, we denote the space translations generator by $P$, the time translations by $H$, and the boosts (or space-time rotations) by $K$. The symbol $I$ stands for the central charge. In this way, gravity-matter interaction is constructed in a manifestly (gauge) tensor form. Cangemi and Jackiw have noticed that the algebra (1) (hereafter denoted "CJ algebra") induces a general non-degenerate symmetric bilinear invariant metric,

$$
\eta=\left(\begin{array}{cccc}
1 & 0 & 0 & 0 \\
0 & -1 & 0 & 0 \\
0 & 0 & a & -1 \\
0 & 0 & -1 & 0
\end{array}\right)
$$


The CJ algebra, as well as the metric $\eta$, was used by Nappi and Witten [5] to construct a conformal field theory describing a homogeneous four dimensional Minkowski space-time based on an ordinary Wess-Zumino-Witten (WZW) action on the non-semisimple algebra (1). The resulting model can be seen as a four dimensional monochromatic plane wave. Shortly after, other authors (see, for instance, $[6,7]$ ) generalized the $\mathrm{CJ}$ algebra to construct classes of exact conformal field theories where the Virasoro central charge is an integer (equal to four in the Nappi-Witten model). This charge is independent of the levels of the corresponding affine Lie algebra and is thus equal to the dimension of the group manifold.

Despite the success of such geometrical approaches in describing different models, the intriguing occurrence of $\eta$, as pointed out to us by Jackiw [4], remains mysterious. Another intriguing aspect mentioned in [3] is that, when diagonalized, the metric (2) gives $\operatorname{diag}(1,-1,1,-1)$, for which the isotropy group is $S O(2,2)$, the conformal group in $(1+1)$ dimensions. The adjoint representation of the $\mathbf{C J}$ group carries a representation for a subgroup of $S O(2,2)$. One purpose here is to analyse the general association of $\eta$ with Lie algebras. The most obvious way to do so is to find Lie algebras that admit $\eta$ as a symmetric bilinear invariant form (of which the Killing form is a particular case). We show that the CJ algebra is not the only algebra for which this is so: the other non-trivial possibility is $s u(1,1) \oplus u(1)$. This result may be of interest in extending the CJ method to other gauge groups with metric $\eta$. On the other hand, we analyse $\eta$ in association with the notion of embedding of metric spaces. Along these lines, we proceed further to explore some aspects of the Galilean symmetries.

In Galilean theories, the metric (2) has been used in various situations in which the space-time is extended to five dimensions. For instance, Künzle et al. [8-11] investigated geometrical aspects of the gravitational Newton-Cartan theory using a five dimensional metric space with a metric tensor of type $\eta$. Then the non-trivially extended Galilei group appears as a subgroup of the affine de Sitter group in $(4+1)$ dimensions. Another instance is that, motivated by the nature of the spontaneous breaking of symmetries in Galilean quantum field theory, Takahashi et al. [12-14] associated a tensor structure with the Galilean transformations, resulting in a geometrical space-time also characterized by $\eta$ in five dimensions. Lie algebraic aspects of such manifolds have been developed in connection with the Lie algebra of the $(4+1)$ de Sitter group and the nature of the embedding of the Galilean $\mathbf{R}^{3} \times \mathbf{R}$ manifold in the $(4+1)$ de Sitter space has been considered [15]. Hereafter, we denote by $\mathscr{G}$ such a five dimensional space (described in Subsection III.A) in which the usual three dimensional Euclidean space is embedded. Besides, in this five dimensional Galilean formalism, attempts have been made to treat the relativistic and Newtonian structures in a unified geometrical way $[11,14]$. This goal has been achieved for relativistic theories by taking $a=c^{2}$ in (2).

However, in this five dimensional formalism, many aspects remain to be explored (for theoretical and practical purposes) that can shed some light on inscrutable non-relativistic processes of condensed matter physics such as, for instance, the 
symmetry breaking in superfluids. For example, phonons, the excitations of the Galilean Schrödinger field, satisfy a wave equation that is invariant under a Lorentz-like set of transformations, where the speed of light is replaced with the speed of sound. On the other hand, despite some attempts, the two non-relativistic limits of the electromagnetic field, such as those derived by Le Bellac and LévyLeblond $[16,17]$, and classical mechanics are not fully accommodated in the five dimensional Galilean formalism. These aspects are important if one anticipates, for instance, the improvement of non-abelian Galilean approaches. In this work, we present an answer for such tasks and, in this scenario, we suggest a Newtonian formulation for the Cangemi-Jackiw gravitation theory. Moreover, aspects of a combined description of the relativistic and non-relativistic theories on a geometrical basis are considered in a new formalism, which in turn rests exclusively on the notion of the embedding of metric spaces. Another aspect we explore here is the development of classical mechanics in a Galilean covariant fashion. One of our results is to derive the gauge structure of classical mechanics, which was pointed out by Elie Cartan and more recently developed by Ghaboussi [18], but proceeding further by including a manifestly Galilean covariant structure.

This article is organized as follows. In Section 2, we set forth the notation and identify the Lie algebras that admit some $\eta$-like metrics as bilinear invariant forms. In Section 3, the Galilei group is revisited under the perspective of the Galilean metric space with metric tensor $\eta$. In Section 4, the electromagnetic tensor is explicitly written in five dimensions and different embeddings are used to derive the two non-relativistic limits of the electromagnetic field. A Galilean non-abelian gauge formalism is also discussed briefly. In Section 5, the aspects of a unified Newtonian and Lorentzian formalism, with the five dimensional space $\mathscr{G}$ used as a starting point, are treated. In Section 6, we present the developments for classical mechanics. Final conclusions and remarks are summarized in Section 7.

\section{BILINEAR FORMS, LIE ALGEBRAS, AND CENTRAL CHARGES}

Let $l=\left\{X_{1}, \ldots, X_{n} ;\left[X_{a}, X_{b}\right]=f_{a b}^{c} X_{c}\right\}$ be a real $n$ dimensional Lie algebra, corresponding to the Lie group $L$, where $f_{a b}^{c}$ are its structure constants. A symmetric bilinear invariant form of $l$ is a bilinear mapping $\Omega: l \times l \rightarrow \mathbf{R}$, which is symmetric

$$
\Omega(X, Y)=\Omega(Y, X)
$$

and invariant under $L$, which means that $\Omega\left(\Phi X \Phi^{-1}, \Phi Y \Phi^{-1}\right)=\Omega(X, Y)$, where $\Phi=\exp (i X) \in L$, for some $X \in l$ (see, for instance, Refs. [19]). This condition can be brought to the form

$$
\Omega(X,[Z, Y])+\Omega(Y,[Z, X])=0 .
$$


With a basis $\left\{X_{1}, X_{2}, \ldots\right\}$ for $l$, the conditions given by (3) and (4) become, respectively,

$$
\Omega_{a, b}=\Omega_{b, a},
$$

where $\Omega_{a, b} \equiv \Omega\left(X_{a}, X_{b}\right)$, and

$$
f_{a b}^{d} \Omega_{c d}+f_{a c}^{d} \Omega_{b d}=0 .
$$

Therefore, given the structure constants $f_{a b}^{c}$ of a Lie algebra $l$, we can find its symmetric bilinear invariant forms by solving (5) and (6) for $\Omega_{a b}$. Conversely, we can consider the problem of finding the Lie algebras that correspond to a given bilinear form. Then one must solve Eq. (6) and the Jacobi identities for the structure constants $f_{a b}^{c}$, knowing $\Omega_{a b}$.

Let us first analyse the Lie algebras that admit the three dimensional metric given by

$$
\Omega \equiv \eta=\left(\begin{array}{ccc}
1 & 0 & 0 \\
0 & 0 & -1 \\
0 & -1 & 0
\end{array}\right),
$$

as a symmetric bilinear invariant form. Such a metric would describe a Galilean covariant theory in a $(1+1)$ space-time. The possible Lie algebras are three dimensional. Let us then denote the basis of the Lie algebras as $\left\{X_{1}, X_{2}, X_{3}\right\}$. The bilinear form $\Omega_{a b} \equiv \Omega\left(X_{a}, X_{b}\right)$ satisfies Eq. (6) with $a, b, c=1,2,3$ and the non-zero elements given by

$$
\Omega_{11}=1, \quad \Omega_{23}=\Omega_{32}=-1 .
$$

Solving Eq. (6) for $f_{a b}^{c}$ we find

$$
f_{13}^{3}=f_{23}^{1}=-f_{12}^{2},
$$

so that

$$
\left[X_{1}, X_{2}\right]=\alpha X_{2}, \quad\left[X_{1}, X_{3}\right]=-\alpha X_{3}, \quad\left[X_{2}, X_{3}\right]=-\alpha X_{1} .
$$

Obviously the solution with $\alpha=0$ leads to the three dimensional abelian algebra, whereas $\alpha \neq 0$ corresponds to $\operatorname{su}(1,1)$, or $A_{3,8}$ according to [20].

Now we analyse the CJ algebra (1), looking for the list of Lie algebras that admit the four dimensional metric (obtained from (2) by setting $a$ equal to zero):

$$
\Omega \equiv \eta=\left(\begin{array}{cccc}
1 & 0 & 0 & 0 \\
0 & 1 & 0 & 0 \\
0 & 0 & 0 & -1 \\
0 & 0 & -1 & 0
\end{array}\right) .
$$


Therefore, the possible Lie algebras are four dimensional, such that a basis can be denoted by $\left\{X_{1}, X_{2}, X_{3}, X_{4}\right\}$. In our formalism, the metric (8) would describe a Galilean covariant theory in a $(2+1)$ space-time. Solving Eq. (6) for $f_{a b}^{c}$, with the bilinear form $\Omega_{a b} \equiv \Omega\left(X_{a}, X_{b}\right)$ given by Eq. (8), we find that $f_{a 1}^{1}, f_{a 2}^{2}, f_{a 3}^{4}$, and $f_{a 4}^{3}$ are all equal to zero, and

$$
\begin{array}{lll}
f_{a 1}^{2}=-f_{a 2}^{1}, & f_{a 1}^{4}=-f_{a 3}^{1}, & f_{a 1}^{3}=-f_{a 4}^{1}, \\
f_{a 2}^{4}=-f_{a 3}^{2}, & f_{a 2}^{3}=-f_{a 4}^{2}, & f_{a 3}^{3}=-f_{a 4}^{4},
\end{array}
$$

for any value of $a=1,2,3,4$. This brings the number of independant structure constants down to four, so that one can write the general commutation relations as

$$
\begin{array}{lll}
{\left[X_{1}, X_{2}\right]=\alpha X_{3}+\beta X_{4},} & {\left[X_{2}, X_{3}\right]=-\beta X_{1}+\delta X_{3},} \\
{\left[X_{1}, X_{3}\right]=\beta X_{2}+\gamma X_{3},} & {\left[X_{2}, X_{4}\right]=-\alpha X_{1}-\delta X_{4},} \\
{\left[X_{1}, X_{4}\right]=\alpha X_{2}-\gamma X_{4},} & {\left[X_{3}, X_{4}\right]=-\gamma X_{1}-\delta X_{2} .}
\end{array}
$$

The corresponding Lie algebras are:

(i) the four dimensional abelian Lie algebra, when $\alpha=\beta=\gamma=\delta=0$;

(ii) the CJ algebra, when $\alpha \neq 0$ and $\beta=\gamma=\delta=0$; and

(iii) $s u(1,1) \oplus u(1)$, for all the other possibilities.

Let us mention that in terms of the classification in [20], the CJ algebra is denoted $A_{4,10}$ and $s u(1,1) \oplus u(1)$ is denoted $A_{3,8} \oplus A_{1}$. That reference might be of interest whenever one needs to consider their subalgebras. Also, let us notice the existence of a central charge in both Lie algebras, CJ and $s u(1,1) \oplus u(1)$.

These results point out the possibility of using not only the CJ group as a gauge group but also the $s u(1,1) \oplus u(1)$, keeping the same metric structure given by $\eta$. In this sense, the CJ Poincaré gauge theory can be generalized for other gauge group generators.

\section{THE METRIC $\eta$ AND GALILEAN COVARIANCE}

In this section we use $\eta$ given by

$$
\eta=\left(\begin{array}{ccc}
h_{i j} & 0 & 0 \\
0 & 0 & -1 \\
0 & -1 & 0
\end{array}\right),
$$

where $h_{i j}$ is the three dimensional Euclidean metric $\left(h_{i j}\right)=\operatorname{diag}(1,1,1)$, in order to analyse the vector space induced by $\eta$ as metric tensor, following the lines and generalizing the earlier results [15]. In particular, we will analyse inhomogeneous isometric transformations and the nature of different embeddings in $\mathscr{G}$. 


\section{A. The Tensor Structure of the Manifold $\mathscr{G}$}

Let $\mathscr{G}$ be a five dimensional metric space, with an arbitrary vector denoted by $x=\left(x^{1}, x^{2}, x^{3}, x^{4}, x^{5}\right)=\left(\mathbf{x}, x^{4}, x^{5}\right)$. The inner product in $\mathscr{G}$ is then defined by

$$
\begin{aligned}
(x \mid y) & =\eta_{\mu v} x^{\mu} y^{v} \\
& =\sum_{i=1}^{n=3} x^{i} y^{i}-x^{4} y^{5}-x^{5} y^{4},
\end{aligned}
$$

where $x, y \in \mathscr{G}$ and $\eta_{\mu \nu}$ is in (10).

Let $\left\{e_{\mu}\right\}=\left\{e_{1}, \ldots, e_{5}\right\}$ be a basis of $\mathscr{G}$, such that $x=x^{\mu} e_{\mu}$ and $y=y^{\mu} e_{\mu}$. Then, from Eq. (11) it follows that $\left(e_{\mu} \mid e_{v}\right)=\eta_{\mu \nu}=\eta_{\nu \mu}$. The dual space $\mathscr{G} *$ is defined by the following 1-forms: $e^{\mu}: x \mapsto x^{\mu}, e^{\mu}(x)=x^{\mu},(x \in \mathscr{G})$. Therefore, for $w \in \mathscr{G}^{*}$, we have $w=w_{\mu} e^{\mu}$ (where $w_{\mu}=w\left(e_{\mu}\right)$ ) and $\left\{e^{\mu}\right\}$ is a basis of $\mathscr{G}^{*}$.

As a result, Eq. (11) can be written as $(x \mid y)=x^{\mu} y_{\mu}=x_{\mu} y^{\mu}$, such that

$$
\begin{aligned}
& e^{i}(x)=x^{i}=x_{i}, \quad i=1,2,3, \\
& e^{4}(x)=x^{4}=-x_{5}, \\
& e^{5}(x)=x^{5}=-x_{4},
\end{aligned}
$$

where we use $\eta_{\mu \nu}$ to raise and lower the indices.

A tensor of type $(n, k)$ is defined by the mapping

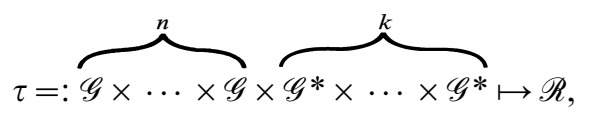

such that $\tau=\tau_{\alpha \beta \ldots \gamma}^{\mu \nu \ldots \sigma} \eta_{\mu v \ldots \sigma}^{\alpha \beta \ldots \gamma}$, where $\tau_{\alpha \beta \ldots \gamma}^{\mu \nu \ldots \sigma}=\tau\left(e^{\mu}, e^{v}, \ldots, e^{\sigma}, e_{\alpha}, e_{\beta}, \ldots, e_{\gamma}\right)$, and

$$
\eta_{\mu \nu \ldots \sigma}^{\alpha \beta \ldots \gamma}=e_{\mu} \otimes e_{\nu} \otimes \cdots \otimes e_{\sigma} \otimes e^{\alpha} \otimes e^{\beta} \otimes \cdots \otimes e^{\gamma}
$$

is a basis for the $(n, k)$ tensor space.

\section{B. Group of Linear Transformations in $\mathscr{G}$}

The set of linear transformations in $\mathscr{G}$ of type $\bar{x}^{\mu}=G_{v}^{\mu} x^{v}+a^{\mu}$ (which leaves $(d x \mid d y)$ invariant), such that $|G|=1$, with $G^{\mu}{ }_{v}=\delta^{\mu}{ }_{v}+\epsilon_{v}^{\mu}$, admits 15 generators of transformations. Using the definition

$$
K_{\alpha}=\left.i \frac{\partial \bar{x}^{\mu}}{\partial \alpha}\right|_{\alpha=0} \frac{\partial}{\partial x^{\mu}}
$$

where $K_{\alpha}$ is the generator associated with the group parameter $\alpha$ (which also labels the group generators), we have 


$$
\begin{aligned}
& J_{3}=-i\left(x^{1} \partial_{2}-x^{2} \partial_{1}\right), \\
& J_{1}=-i\left(x^{2} \partial_{3}-x^{3} \partial_{2}\right), \\
& J_{2}=-i\left(x^{3} \partial_{1}-x^{1} \partial_{3}\right), \\
& G_{i}=i\left(x^{4} \partial_{i}+x^{i} \partial_{5}\right), \\
& C_{i}=i\left(x^{5} \partial_{i}+x^{i} \partial_{4}\right), \\
& D=i\left(x^{4} \partial_{4}-x^{5} \partial_{5}\right), \\
& P_{\mu}=i \partial_{\mu}
\end{aligned}
$$

where $i=1,2,3$ and $\mu=1,2, \ldots, 5$. These generators satisfy the commutation relations

$$
\begin{aligned}
{\left[M_{\mu v}, M_{\rho \sigma}\right] } & =-i\left[\eta_{v \rho} M_{\mu \sigma}-\eta_{\mu \rho} M_{v \sigma}+\eta_{\mu \sigma} M_{v \rho}-\eta_{v \sigma} M_{\mu \rho}\right] \\
{\left[P_{\mu}, M_{\rho \sigma}\right] } & =-i\left[\eta_{\mu \rho} P_{\sigma}-\eta_{\mu \sigma} P_{\rho}\right] \\
{\left[P_{\mu}, P_{v}\right] } & =0
\end{aligned}
$$

where $M_{\alpha \beta}(\alpha, \beta=1, \ldots, 5)$ are defined by

$$
\begin{aligned}
& M_{i j}=-M_{j i}=\varepsilon_{i j k} J_{k}, \\
& M_{5 i}=-M_{i 5}=G_{i}, \\
& M_{4 i}=-M_{i 4}=C_{i}, \\
& M_{54}=-M_{45}=D .
\end{aligned}
$$

The commutation relations (20)-(22) define an inhomogeneous Lorentz algebra in $(4+1)$ dimensions, which is therefore analogous to the Poincaré algebra. We denote this algebra by $g$.

A particular subalgebra of $g$ is given by

$$
\begin{gathered}
{\left[L_{i}, L_{j}\right]=i \varepsilon_{i j k} L_{k}, \quad\left[L_{i}, P_{j}\right]=i \varepsilon_{i j k} P_{k}, \quad\left[L_{i}, B_{j}\right]=i \varepsilon_{i j k} B_{k},} \\
{\left[B_{i}, P_{4}\right]=i P_{i}, \quad\left[B_{i}, P_{j}\right]=i P_{5} \delta_{i j} .}
\end{gathered}
$$

These Lie brackets are just the Galilei-Lie algebra with the usual central charge $P_{5}$. This suggests that we have embedded the Euclidean space $\mathscr{E}$ in the five dimensional $\mathscr{G}$.

It is well known that the subset of the Poincare transformations that leave a light-like vector invariant forms a Galilean subgroup. This is analogous to our de Sitter space and the non-homogeneous group transformations. But if we consider only the Poincare group, and its isometric underlying space-the Minkowski space - we will not derive the full covariance that we have derived. Actually, in our 
approach, if we start with the Poincare group (or the Minkowski space) we can derive no more than a two dimensional Galilean physics.

C. Embeddings of $\mathscr{E}$ in $\mathscr{G}$

In the present section, we consider three possible embeddings of $\mathscr{E}$ in $\mathscr{G}$, useful for our purposes.

(i) The first embedding is [12]

$$
\mathfrak{I}_{1}: \mathbf{A} \mapsto A=\left(\mathbf{A}, A_{4}, \frac{\mathbf{A}^{2}}{2 A_{4}}\right), \quad \text { where } \quad \mathbf{A}=\left(A^{1}, A^{2}, A^{3}\right) \in \mathscr{E}, A \in \mathscr{G} .
$$

It follows that $A$ is null-like, since

$$
\begin{aligned}
(A \mid A) & =\eta_{\mu v} A^{\mu} A^{v}, \\
& =\sum_{i=1}^{3} A^{i} A^{i}-2 A^{4} A^{5}=0 .
\end{aligned}
$$

That is, according to $\mathfrak{J}_{1}$, each vector in $\mathscr{E}$ is in homomorphic correspondence with null-like vectors in $\mathscr{G}$. An example of this type of vector is given by $x=$ $\left(\mathbf{x}, k t, \mathbf{x}^{2} / 2 k t\right)$, where $k$ is some constant with units of velocity. Under the group defined by the generators of the Lie algebra given by Eq. (27) (considering a nonunitary representation), that is,

$$
K_{i}=e^{-v^{i} B_{i}}, \quad R_{i j}=e^{\epsilon_{i j k} L_{k}}, \quad T_{i}=e^{a^{i} P_{i}}, \quad T_{4}=e^{b H},
$$

where $a^{5}=0, H=P^{4}$, the vector $x=\left(\mathbf{x}, k t, \mathbf{x}^{2} / 2 k t\right)$ is transformed as

$$
\begin{aligned}
& \bar{x}^{i}=R_{j}^{i} x^{j}-v^{i} x^{4}+\mathbf{a}, \\
& \bar{x}^{4}=x^{4}+b, \\
& \bar{x}^{5}=x^{5}-v^{i}\left(L_{j}^{i} x^{j}\right)+\frac{1}{2} \mathbf{v}^{2} x^{4} .
\end{aligned}
$$

(ii) A second embedding is

$$
\mathfrak{I}_{2}: \mathbf{A} \mapsto A=\left(\mathbf{A}, A_{4}, 0\right) .
$$

Then $A$ is no longer a null-like vector, for $(A \mid A)=\mathbf{A}^{2}$. An example is the vector $x=\left(\mathbf{x}, x^{4}, 0\right), x^{4}=k t$.

(iii) A third possible embedding is

$$
\mathfrak{I}_{3}: \mathbf{A} \mapsto A=\left(\mathbf{A}, \frac{A_{4}}{\sqrt{2}}, \frac{A_{4}}{\sqrt{2}}\right) .
$$

Then we have $(A \mid A)=\mathbf{A}^{2}-\left(A_{4}\right)^{2}$. Therefore, the embedding is a Minkowskian plane in $\mathscr{G}$. 


\section{IV. $\mathscr{G}$-COVARIANCE AND THE ELECTROMAGNETIC FIELD}

Let us assume that in $\mathscr{G}$ we have the following set of Maxwell's equations,

$$
\begin{gathered}
\mathrm{d} F=0, \\
\mathrm{~d}^{*} F={ }^{*} ;
\end{gathered}
$$

where ${ }^{*} F=1 / 2 \omega(F),{ }^{*} j=\omega(j), \omega=\mathrm{d} x^{1} \wedge \mathrm{d} x^{2} \wedge \mathrm{d} x^{3} \wedge \mathrm{d} x^{4} \wedge \mathrm{d} x^{5}$ is a volume form, such that $F=\mathrm{d} A$, where $A$ is the gauge potential. Let us write $F$ in terms of its components as the antisymmetric matrix

$$
\left(F_{\mu v}\right)=\left[\begin{array}{ccccc}
0 & b_{3} & -b_{2} & c_{1} & d_{1} \\
-b_{3} & 0 & b_{1} & c_{2} & d_{2} \\
b_{2} & -b_{1} & 0 & c_{3} & d_{3} \\
-c_{1} & -c_{2} & -c_{3} & 0 & 0 \\
-d_{1} & -d_{2} & -d_{3} & 0 & 0
\end{array}\right]
$$

and $j=\left(\mathbf{j}, j_{4}, j_{5}\right)$, a five dimensional current. From (33) and (34), we obtain

$$
\begin{array}{rlrl}
\nabla \cdot \mathbf{b} & =0, & & \nabla \times \mathbf{b}-2 \partial_{4} \mathbf{d}=\mathbf{j}, \\
\nabla \times \mathbf{c}+\partial_{4} \mathbf{b}=0, & \nabla \cdot \mathbf{d}=j^{4}, \\
\nabla \times \mathbf{d}+\partial_{5} \mathbf{b}=0, & \nabla \cdot \mathbf{c}=j^{5},
\end{array}
$$

where $\mathbf{c}=\left(c_{1}, c_{2}, c_{3}\right)$ and $\mathbf{d}=\left(d_{1}, d_{2}, d_{3}\right)$. In this section, we interpret the elements of the antisymetric tensor in (35) to be $\mathbf{b} \rightarrow \mathbf{B}$, the magnetic field; $\mathbf{c}=0 ; \mathbf{d} \rightarrow \mathbf{E}$, the electric field; and 5-vector $j \rightarrow(\mathbf{J}, \rho, 0)$, where $\mathbf{J}$ is the electric-current vector and $\rho$ is the charge distribution (a different interpretation is considered in the following section). However, in order to derive some physics, one must also specify the embedding of the space-time in $\mathscr{G}$; and, as we saw in the last section, different possibilities are available. Two of them are analysed in the following, resulting in the two non-relativistic limits for the electromagnetic field.

\section{A. Magnetic Non-relativistic Limit of Maxwell's Equations}

In this section, using the present covariant formalism, we derive the nonrelativistic limit of the Maxwell equations called the "magnetic limit." Let us proceed then by assuming the embedding $\mathscr{I}: \mathbf{x} \rightarrow x=\left(\mathbf{x}, x^{4}, x^{5}\right)=(\mathbf{x}, 0, c t$ ) (in order to adjust the units of the embedded vector in $\mathscr{G}$, we have used the speed of light in the coordinate $x_{5}=c t$ ), such that, under the Galilean boost, characterized by $\mathbf{v}$, we have

$$
\overline{\mathbf{x}}=\mathbf{x}, \quad \bar{x}^{4}=0, \quad \bar{t}=t+\frac{\mathbf{v}}{c^{2}} \cdot \mathbf{x} .
$$


Under these conditions, we derive from (36)

$$
\begin{aligned}
\nabla \cdot \mathbf{E} & =\rho, & \nabla \cdot \mathbf{B} & =0 \\
\nabla \times \mathbf{E}+\partial_{t} \mathbf{B} & =0, & \nabla \times \mathbf{B} & =\mathbf{J} .
\end{aligned}
$$

Such a set of equations, invariant under the Galilean transformations given by (37), is the Maxwell equations in the magnetic limit, specified according to Le Bellac and Lévy-Leblond by $v / c \ll 1,\|c t\| \ll\|\mathbf{x}\|$, and $\|\mathbf{E}\| \ll c\|\mathbf{B}\|[17]$.

\section{B. Electric Non-relativistic Limit of Maxwell's Equations}

The second case, the "electric limit," is obtained if we consider the embedding $\mathscr{I}: \mathbf{x} \rightarrow x=\left(\mathbf{x}, x^{4}, x^{5}\right)=(\mathbf{x}, c t, 0)$, such that, under the Galilean boost, we have

$$
\overline{\mathbf{x}}=\mathbf{x}+\mathbf{v} t, \quad \bar{x}^{4}=\bar{t}=t, \quad \bar{x}^{5}=0 .
$$

Therefore, from (36) we derive

$$
\begin{aligned}
\nabla \cdot \mathbf{E} & =\rho, & \nabla \cdot \mathbf{B} & =0 \\
\nabla \times \mathbf{E} & =0, & \nabla \times \mathbf{B}-\partial_{t} \mathbf{E} & =\mathbf{J},
\end{aligned}
$$

which are the Maxwell equations in the electric non-relativistic limit, characterized according to Le Bellac and Lévy-Leblond by $v / c \ll 1,\|\mathbf{x}\| \ll\|c t\|$, and $c\|\mathbf{B}\| \ll\|\mathbf{E}\|\left[\right.$ [17]. (Note that in (42), $\mathbf{J}$ has been replaced by $\mathbf{J}+\partial_{t} \mathbf{E}$.)

Therefore, through these different embeddings, we have derived both nonrelativistic limits of the electromagnetic field from the five dimensional covariant structure. This fact opens the possibility of carrying out a proper analysis for the non-abelian fields in Galilean space-time.

\section{Non-abelian Theories}

Let us assume in $\mathscr{G}$ the form

$$
\begin{aligned}
\mathrm{d} F & =\mathrm{d} A+A \wedge A, \\
& =\eta^{\mu \sigma} \eta^{v \rho} F_{\mu \nu}^{a} X_{a} \mathrm{~d} x_{\sigma} \wedge \mathrm{d} x_{\rho}
\end{aligned}
$$

where $X_{a}$ is an element of the gauge group, and the gauge potential is

$$
A=\eta^{\mu v} A_{\mu}^{a} X_{a} \mathrm{~d} x_{v} .
$$

It is clear from (43) and (44) that, as pointed out by Künzle and Duval [11], we can derive a non-abelian theory in a Galilean covariant form by replacing the flatspace Minkowski metric in the usual theory by $\eta^{\mu \nu}$. Nevertheless, we need to specify the embedding; the embeddings analysed previously to obtain the two nonrelativistic limits of the electromagnetic field are natural candidates. We do not intend to go much further here with non-abelian theories, which will be the subject of another article. However, it is worth noting that a Galilean CJ theory can be 
derived if in (43) and (44) we consider $\eta^{\mu v}$ as given by Eq. (10) with $h_{i j}=1$ and $X_{a}$, the generator of the CJ algebra (1), resulting in an expression that is formally the one derived by Cangemi and Jackiw [3], that is,

$$
\begin{aligned}
F & =F^{a} X_{a} \\
& =\left(\mathrm{d} a+\varepsilon_{b}^{a} \omega e^{b}\right) P_{a}+\mathrm{d} \omega K+\left(\mathrm{d} a+\frac{1}{2} e^{a} \varepsilon_{a b} e^{b}\right) I,
\end{aligned}
$$

where the gauge curvature associated with the translation generators describes a torsion density, while for the generators of boost gauge curvature, it is the scalar curvature density [3].

\section{V. $\mathscr{G}$-MANIFOLD AND LORENTZ-LIKE INVARIANT THEORIES}

In the present section, we show how to use the five dimensional $\mathscr{G}$-manifold to derive, via a different embedding, Lorentz-like invariant approaches. First, we use the embedding given by Eq. (32), such that

$$
\mathbf{x} \rightarrow x=\left(\mathbf{x}, \frac{k t}{\sqrt{2}}, \frac{k t}{\sqrt{2}}\right)
$$

and

$$
\partial=\left(\partial_{i}=\frac{\partial}{\partial x^{i}}, \quad \partial_{4}=\frac{1}{\sqrt{2}} \frac{\partial}{k \partial t}, \quad \partial_{5}=\frac{1}{\sqrt{2}} \frac{\partial}{k \partial t}\right) .
$$

This leads to a Minkowskian invariant of the type $d^{2}=\mathbf{x}^{2}-k^{2} t^{2}$.

Let us then consider, as an example, $k=c(=1)$ in the case of the electromagnetic field. We define in Eq. (36) $\mathbf{b}=\mathbf{B}$ as the magnetic field, $\mathbf{c}=\mathbf{d}=\mathbf{E} / \sqrt{2}$ as the electric field, and $j=(\mathbf{J}, \rho / \sqrt{2}, \rho / \sqrt{2})$, where $\mathbf{J}$ is the electric current and $\rho$ is the charge distribution. From this, one finds the Maxwell equations $\nabla \cdot \mathbf{B}=0$, $\nabla \times \mathbf{E}+\partial_{t} \mathbf{B}=0, \nabla \times \mathbf{B}-\partial_{t} \mathbf{E}=\mathbf{J}$, and $\nabla \cdot \mathbf{E}=\rho$.

This procedure also works for other fields. Indeed, considering the Casimir invariant $\partial_{\mu} \partial^{\mu}$ of the algebra given by (20)-(22), we write

$$
\partial_{\mu} \partial^{\mu} \psi=n^{2} \psi
$$

where $n$ is a fixed value of the Casimir invariant for each specific representation. Using the embedding defined in (45) and (46), it follows that

$$
\left(\nabla^{2}-\frac{\partial^{2}}{k^{2} \partial t^{2}}-n^{2}\right) \psi=0
$$


Observe that Eq. (48) is a Lorentz-like invariant but does not necessarily describe a relativistic theory. As a matter of fact, we have different possibilities according to the choice of the constants $k$ and $n$. For instance, when $k=c$ (the speed of light) and $n=m$ (the mass of a bosonic particle), then Eq. (48) is the Klein-Gordon equation. On the other hand, for $k=c_{s}$, the speed of sound, and $n=0$, Eq. (48) can be interpreted as the phonon wave equation. This mechanism of embedding can then explain, from a geometrical standpoint, why we can find Lorentz-like symmetries in the scenario of Galilean theories, as is the case of the wave equations in matter. This aspect leads us to the conjecture about the existence of a Dirac-like equation for non-relativistic physics, in the following sense.

A linearized version of (47) is the equation

$$
\left(\gamma^{\mu} \partial_{\mu}+n\right) \Psi=0
$$

where the five matrices $\gamma^{\mu}$ are given by [14]

$$
\gamma^{i}=\left(\begin{array}{cc}
\sigma^{i} & 0 \\
0 & -\sigma^{i}
\end{array}\right), \quad \gamma^{4}=\left(\begin{array}{cc}
0 & 0 \\
-\sqrt{2} & 0
\end{array}\right), \quad \gamma^{5}=\left(\begin{array}{cc}
0 & \sqrt{2} \\
0 & 0
\end{array}\right),
$$

so that $\gamma^{\mu} \partial_{\mu}$ is invariant (where $\sigma^{i}(i=1,2,3)$ are the $(2 \times 2)$ Pauli matrices). The matrices $\gamma^{\mu}$ satisfy the Clifford algebra

$$
\left\{\gamma^{\mu}, \gamma^{\nu}\right\}=2 \eta^{\mu \nu}
$$

Therefore, Eq. (49) is $g$-invariant. Using the embedding given in (45) and (46), Eq. (49) reduces to

$$
\left(\gamma^{1} \partial_{1}+\gamma^{2} \partial_{2}+\gamma^{3} \partial_{3}+\frac{1}{k \sqrt{2}}\left(\gamma^{4}+\gamma^{5}\right) \partial_{t}+n\right) \Psi=0,
$$

resulting in the usual form of the Dirac equation (in a four dimensional space), that is,

$$
\left(\gamma^{\mu} \partial_{\mu}+n\right) \Psi=0
$$

where $\mu=1,2,3,4$. The metric has a Minkowski signature, $(+++-), \gamma^{i}$ are given in Eq. $(50)$, and $(1 / \sqrt{2})\left(\gamma^{4}+\gamma^{5}\right) \rightarrow \gamma^{4}$, which is the matrix

$$
\gamma^{4}=\left(\begin{array}{cc}
0 & 1 \\
-1 & 0
\end{array}\right)
$$

Then, if $n=m$, the mass of a spin $1 / 2$ particle, and $k=c$, Eq. (53) is the Dirac equation. But notice that this is not the only possibility, as is the case for the scalar field. The interpretation of (49), for general values of $n$, has not been explored yet. 


\section{VI. $\mathscr{G}$-COVARIANT CLASSICAL MECHANICS}

We define a 10 dimensional phase space as a manifold $\Gamma$ defined via the symplectic 2-form

$$
w=\eta^{\mu v} \mathrm{~d} q_{\mu} \wedge \mathrm{d} p_{v}, \quad \mu=1,2, \ldots, 5,
$$

and by a vector field,

$$
X_{f}=\frac{\partial f}{\partial p_{\mu}} \frac{\partial}{\partial q^{\mu}}-\frac{\partial f}{\partial q^{\mu}} \frac{\partial}{\partial p_{\mu}},
$$

where $f$ is a $C^{\infty}$ function in $\Gamma$, such that a Poisson bracket $\{f, g\}$ is introduced by

$$
\begin{aligned}
w\left(X_{f}, X_{h}\right) & =\{f, h\} \\
& =d q\left(X_{f}\right) d p\left(X_{h}\right)-d p\left(X_{f}\right) d q\left(X_{h}\right), \\
& =\eta^{\mu v}\left(\frac{\partial f}{\partial q^{\mu}} \frac{\partial g}{\partial p^{v}}-\frac{\partial g}{\partial q^{\mu}} \frac{\partial f}{\partial p^{v}}\right) .
\end{aligned}
$$

On the other hand, since $w\left(X_{h}\right)=\mathrm{d} h$, we have $\{f, h\}=\mathrm{d} f\left(X_{h}\right)=\left\langle\mathrm{d} f, X_{h}\right\rangle$. Then $\left\langle\mathrm{d} f, X_{\{h, g\}}\right\rangle+\left\langle\mathrm{d} h, X_{\{g, f\}}\right\rangle+\left\langle\mathrm{d} g, X_{\{f, h\}}\right\rangle=0$, such that $\left[X_{h}, X_{f}\right]=X_{\{f, h\}}$, inducing a representation of Lie groups. Considering the $g$-Lie algebra it can be shown that

$$
\begin{aligned}
{\left[X_{M_{\mu v}}, X_{M_{\rho \sigma}}\right] } & =-\eta_{v \rho} X_{M_{\mu \sigma}}+\eta_{\mu \rho} X_{M_{v \sigma}}-\eta_{\mu \sigma} X_{M_{v \rho}}+\eta_{v \sigma} X_{M_{\mu \rho}}, \\
{\left[X_{P_{\mu}}, X_{M_{\rho \sigma}}\right] } & =-\eta_{\mu \rho} X_{P_{\sigma}}+\eta_{\mu \sigma} X_{P_{\rho}}, \\
{\left[X_{P_{\mu}}, X_{P_{\rho}}\right] } & =0
\end{aligned}
$$

where $X_{M_{\mu \nu}}=-\left\{M_{\mu \nu},\right\}$, and $M_{\mu v}=q_{\mu} p_{v}-p_{\nu} q_{\mu}$, with

$$
\begin{aligned}
& M_{i j}=q^{i} p_{j}-p_{i} q^{j}, \\
& M_{5 i}=q^{4} p_{i}-p_{5} q^{i}, \\
& M_{4 i}=q^{5} p_{i}-p_{4} q^{i}, \\
& M_{54}=q^{4} p_{4}-p_{5} q^{5} .
\end{aligned}
$$

It is possible to derive a classical mechanics theory assuming a flow such that

$$
\partial_{\mu} f=-X_{p_{\mu}} f
$$

where $f(q, p)$ is a real $\left(C^{\infty}\right)$ density distribution function in $\Gamma$, and the (five dimensional) vectors $q$ and $p$ are defined through the embedding given by Eq. (28), that 
is, $q=\left(\mathbf{q}, t, \mathbf{q}^{2} / 2 t\right)$ and $p=(\mathbf{p}, H, m)$, where $H=\mathbf{p}^{2} / 2 m$. Then, in terms of components, we have from Eq. (59)

$$
\begin{aligned}
& \partial_{i} f=-X_{p_{i}} f \mapsto \partial_{i} f=\left\{p_{i}, f\right\}, \\
& \partial_{4} f=-X_{p_{4}} f \mapsto \partial_{t} f=\{H, f\}, \\
& \partial_{5} f=-X_{p_{5}} f \mapsto \partial_{5} f=0 .
\end{aligned}
$$

Consistency relations are given by Eqs. (60) and (62), whereas Eq. (61) is nothing but the Liouville equation. Notice that this derivation has been possible because of the nature of the embedding. In this case, the symplectic structure defined in Eq. (55) reduces to

$$
w=\mathrm{d} q_{i} \wedge \mathrm{d} p^{i}-\mathrm{d} t \wedge \mathrm{d} H,
$$

and from Eq. (59) we can write $D_{\mu} f=0$, where $D_{\mu}=\partial_{\mu}+X_{p_{\mu}}$, that is,

$$
\begin{aligned}
& D_{i}=D_{5}=0, \quad i=1,2,3, \\
& D_{t}=\partial_{t}+X_{H} .
\end{aligned}
$$

Equations (63) and (65) provide the gauge structure of classical mechanics, as explored by Ghaboussi [18].

\section{CONCLUDING REMARKS}

To summarize, we have explored several consequences of Galilean covariance using a metric similar to $\eta$ (Eq. (2):

(i) Depending on the dimension, we have shown that there are different Lie algebras associated with $\eta$. For instance, in four dimensions, we have derived not only the Cangemi-Jackiw Lie algebra, Eq. (1), but also $s u(1,1) \oplus u(1)$;

(ii) we have used $\eta$, as a metric tensor, to derive Galilean as well as Lorentzian theories in a geometrically unified way, exploring the notion of embeddings of the Euclidean space in the space $\mathscr{G}$, a five dimensional space with metric $\eta$;

(iii) through a five dimensional representation of the equations $\mathrm{d} F=0$ and $\mathrm{d}^{*} F=* j$, we have provided the two non-relativistic limits of the electromagnetic field in a covariant form;

(iv) we have discussed Galilean non-abelian theories, in particular the Poincaré-gauge theory proposed by Cangemi and Jackiw;

(v) we have derived the inhomogeneous transformation group in $\mathscr{G}$; and

(vi) we have introduced a canonical classical representation resulting in a gauge structure associated to a generalized symplectic form. 
The results derived here set forth the basis for other systematic studies in the context of Galilean non-abelian theories and classical mechanics under a covariant perspective.

\section{ACKNOWLEDGMENTS}

We are grateful to Professor R. Jackiw for suggesting this study, Professor H. P. Künzle for discussions and bringing to our attention the references [8-11], Professor Y. Takahashi for his interest in this work, Dr. R. K. Teshima for verification of some results, and Professor C. Nunes for a careful reading of the manuscript. The NSERC of Canada and CAPES and CNPq of Brazil are acknowledged for financial support.

\section{REFERENCES}

1. D. Cangemi and R. Jackiw, Phys. Rev. Lett. 69 (1992), 233.

2. D. Cangemi and R. Jackiw, Phys. Lett. B 299 (1993), 24.

3. D. Cangemi and R. Jackiw, Ann. Phys. (N.Y.) 225 (1993), 229.

4. R. Jackiw, private communication.

5. C. R. Nappi and E. Witten, Phys. Rev. Lett. 71 (1993), 3751; E. Witten, Comm. Math. Phys. 92 (1984), 455.

6. D. I. Olive, E. Rabinovici, and A. Schwimmer, Phys. Lett. B 321 (1994), 361.

7. K. Sfetsos, Phys. Lett. B 324 (1994), 335.

8. C. Duval, G. Burdet, H. P. Künzle, and M. Perrin, Phys. Rev. D 31 (1985), 1841.

9. H. P. Künzle, Can. J. Phys. 64 (1986), 185.

10. H. P. Künzle and C. Duval, Class. Quantum Grav. 3 (1986), 957.

11. H. P. Künzle and C. Duval, in "Semantical Aspects of Spacetime Theories" (U. Majer and H. J. Schmidt, Eds.), p. 113, BI-Wissenschaftsverlag, Mannheim, 1994.

12. Y. Takahashi, Fortschr. Phys. 36 (1988), 63.

13. Y. Takahashi, Fortschr. Phys. 36 (1988), 83.

14. M. Omote, S. Kamefuchi, Y. Takahashi, and Y. Ohnuki, Fortschr. Phys. 37 (1989), 933.

15. A. E. Santana, F. C. Khanna, and Y. Takahashi, Prog. Theor. Phys. 99 (1998), 327.

16. J. M. Lévy-Leblond, Comm. Math. Phys. 6 (1967), 286.

17. M. Le Bellac and J. M. Lévy-Leblond, Nuovo Cimento B 14 (1973), 217.

18. F. Ghaboussi, J. Math. Phys. 34 (1993), 4000.

19. J. Humphreys, "Introduction to Lie Algebras and Representation Theory," SpringerVerlag, New York, 1972; N. Jacobson, "Lie algebras," Wiley-Interscience, New York, 1962.

20. J. Patera and P. Winternitz, J. Math. Phys. 18 (1977), 1449. 